\title{
The Radio Properties of Type Ibc Supernovae
}

\author{
Alicia M. Soderberg \\ Caltech Astronomy, MC 105-24, Pasadena, CA 91106, USA
}

\begin{abstract}
Over the past few years, long-duration $\gamma$-ray bursts (GRBs), including the subclass of Xray flashes (XRFs), have been revealed to be a rare variety of Type Ibc supernova (SN Ibc). While all these events result from the death of massive stars, the electromagnetic luminosities of GRBs and XRFs exceed those of ordinary Type Ibc SNe by many orders of magnitude. The observed diversity of stellar death corresponds to large variations in the energy, velocity, and geometry of the explosion ejecta. Using multi-wavelength (radio, optical, X-ray) observations of the nearest GRBs, XRFs, and SNe Ibc, I show that while GRBs and XRFs couple at least $\sim 10^{48}$ erg to relativistic material, $\mathrm{SNe}$ Ibc typically couple $\lesssim 10^{48}$ erg to their fastest (albeit non-relativistic) outflows. Specifically, I find that less than 3\% of local SNe Ibc show any evidence for association with a GRB or XRF. Recently, a new class of GRBs and XRFs has been revealed which are under-luminous in comparison with the statistical sample of GRBs. Owing to their faint high-energy emission, these sub-energetic bursts are only detectable nearby $(z \lesssim 0.1)$ and are likely 10 times more common than cosmological GRBs. In comparison with local SNe Ibc and typical GRBs/XRFs, these explosions are intermediate in terms of both volumetric rate and energetics. Yet the essential physical process that causes a dying star to produce a GRB, XRF, or sub-energetic burst, and not just a SN, remains a crucial open question. Progress requires a detailed understanding of ordinary SNe Ibc which will be facilitated with the launch of wide-field optical surveys in the near future.
\end{abstract}

Keywords: Stars: Supernovae

PACS: $97.60 . \mathrm{Bw}$

\section{INTRODUCTION}

The discovery of several gamma-ray bursts (GRBs) and X-ray flashes (XRFs) at $z \lesssim 0.3$ in the last few years has firmly established that GRBs and XRFs are accompanied by supernovae of Type Ibc (SNe Ibc) (see Woosley and Bloom 1 for a review). While the distribution of GRB/XRF-SN optical luminosities appears indistinguishable from that of local SNe Ibc [2], spectroscopy reveals unusually broad absorption lines ("broad-lined", BL) in every case [3]. At other wavelengths, these explosions are easily distinguished since GRBs and XRFs produce mildly-relativistic ejecta, which gives rise to strong nonthermal "afterglow" emission. Radio observations are critical in this analysis since they provide the best calorimetry of the fastest ejecta.

Recently, we have identified a population of GRBs/XRFs that are sub-energetic by a factor of $\sim 10^{2}$ and about 10 times more common than typical bursts [4,5]. Given their under-luminous prompt energy release, current satellites can only detect them nearby $(z \lesssim 0.1$ ). These sub-energetic explosions are intermediate between GRBs/XRFs and local SNe Ibc with respect to gamma-ray energy, jet collimation, and volumetric rate, and thus hint at an overall continuum.

Motivated by the GRB/XRF-SN connection and the discovery of sub-energetic GRBs, I embarked on a survey of optically-selected local $(d \lesssim 200 \mathrm{Mpc})$ SNe Ibc with the 


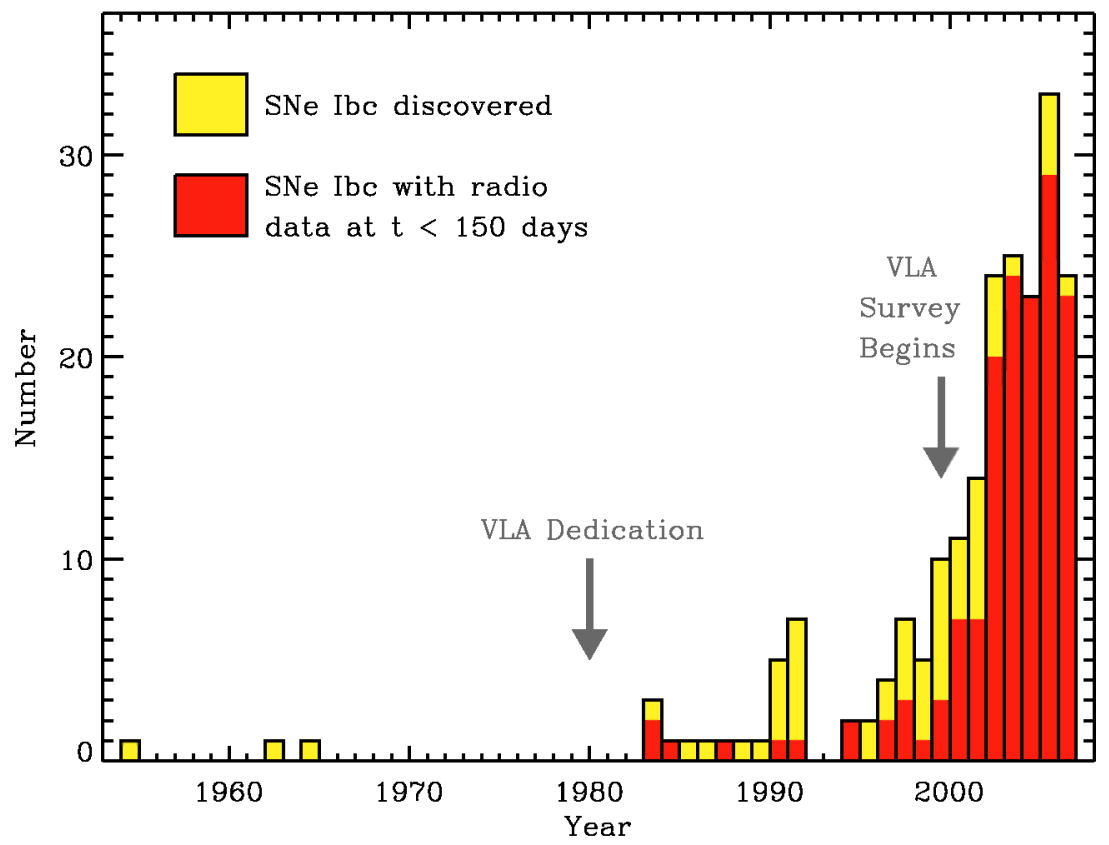

FIGURE 1. The discovery rate of SNe Ibc each year (yellow) is compared with the fraction observed in the radio on timescales less than 150 days (red). Early radio observations are crucial for constraining underluminous and/or off-axis GRBs since they trace the fastest ejecta in the explosion. Since the launch of our VLA survey, nearly every SN Ibc has been observed on this timescale.

Very Large Array (VLA). The goal is to determine the fraction of SNe Ibc that produce mildly-relativistic ejecta. This survey is well-suited to recognizing bursts for which no gamma-ray emission is detected, either because the jets are pointed away from our line-of-sight ("off-axis") or the emission is below the detection thresholds of current satellites.

Since then I have observed $\sim 200$ SNe Ibc with the VLA, on timescales of days to years after the explosion. This dedicated effort has served to characterize in a systematic way the environments and ejecta properties of SNe Ibc for the first time. Early radio observations are crucial for identifying sub-energetic and/or uncollimated explosions. On the other hand, late-time radio observations are sensitive to jets initially beamed away from our line-of-sight. Through this intense VLA program, I have established that (i) roughly $15 \%$ of $\mathrm{SNe}$ Ibc show detectable radio emission, (ii) less than $\sim 3 \%$ show radio luminosities comparable to those observed for GRB/XRF afterglows [6, 5], (iii) less than $10 \%$ of SNe Ibc harbor typical GRBs pointed away from our line-of-sight $[7,8]$, and (iv) basic optical properties (peak luminosity, photospheric velocities) are not reliable indicators of strong radio emission and/or relativistic ejecta. 


\section{A LARGE RADIO SURVEY OF SNE IBC: THE SAMPLE}

Beginning in 2002, I have obtained radio observations for every newly discovered SN Ibc within a maximum distance of $\sim 200 \mathrm{Mpc}$ and accessible to the VLA. All targets are drawn from astronomical circulars which typically report $\sim 20$ new SNe Ibc each year, of which $\sim 90 \%$ are accessible to the VLA. Almost all of the reported SNe Ibc are found through "targeted" SN search campaigns which monitor only the most luminous local galaxies (e.g., RC3 catalog).

Upon spectral classification, I immediately trigger a VLA Target-of-Opportunity observation resulting in first epoch radio observations within a few days of discovery. Since most SNe Ibc are discovered near maximum light, the first epoch VLA observations correspond to days to weeks after the explosion. Follow-up observations for each SN are scheduled logarithmically in time since the explosion.

I supplement this sample with radio data of SNe Ibc taken before 2002. The majority of these data were extracted from the VLA archive and were primarily taken for radio studies of nearby galaxies, while some were taken specifically for SN follow-up. As a result, the archival data generally probe significantly later timescales than my VLA survey. This is highlighted in Figure 1 where the discovery rate of SNe Ibc is compared with the fraction observed with the VLA; early observations ( $t \lesssim 150$ days) were uncommon in the years preceding my VLA survey.

The combined dataset includes all VLA observations of local SNe Ibc to date.

\section{THE RADIO PROPERTIES OF SNE IBC}

This large sample of radio observations are presented in detail in Soderberg [9]. The large majority of these observations were conducted at 4.86 and/or $8.46 \mathrm{GHz}$. As shown in Figure 2, the fraction of SNe Ibc with detectable radio emission is small, $\sim 15 \%$, corresponding to a total of $30 \mathrm{SNe} \mathrm{Ibc}$ detected to date.

Of those with positive detections, the majority $(\sim 70 \%)$ are discovered before radio maximum with well-constrained spectral peaks. As shown in Figures 2 and 3 , the peak radio luminosities span four orders of magnitude, $L_{v \text {,radio }} \approx 10^{26}-10^{30} \mathrm{erg} \mathrm{s}^{-1} \mathrm{~Hz}^{-1}$ on timescales of 1 to 1000 days. Figure 2 shows that GRB/XRF-SNe are distinguished from optically-selected SNe Ibc by their strong, early-peaking radio emission. As discussed in the next section, these properties can be used as a proxy for the presence of relativistic ejecta.

Normalizing by the distance to which each SN could be detected and the effective monitoring time of the survey, we produce the radio luminosity function shown in Figure 3. Clearly, sub-luminous radio SNe Ibc are the most common, though least often detected.

As discussed in detail in Soderberg [9], I find no evidence for any correlation between basic optical properties (peak luminosity, photospheric velocities) and radio luminosity. This holds, in particular, for the fraction of broad-lined SNe Ibc for which the radio detection rate is no higher than that observed for ordinary SNe Ibc. I therefore emphasize that neither optical luminosity nor BL spectral features are reliable proxies for strong radio emission. 


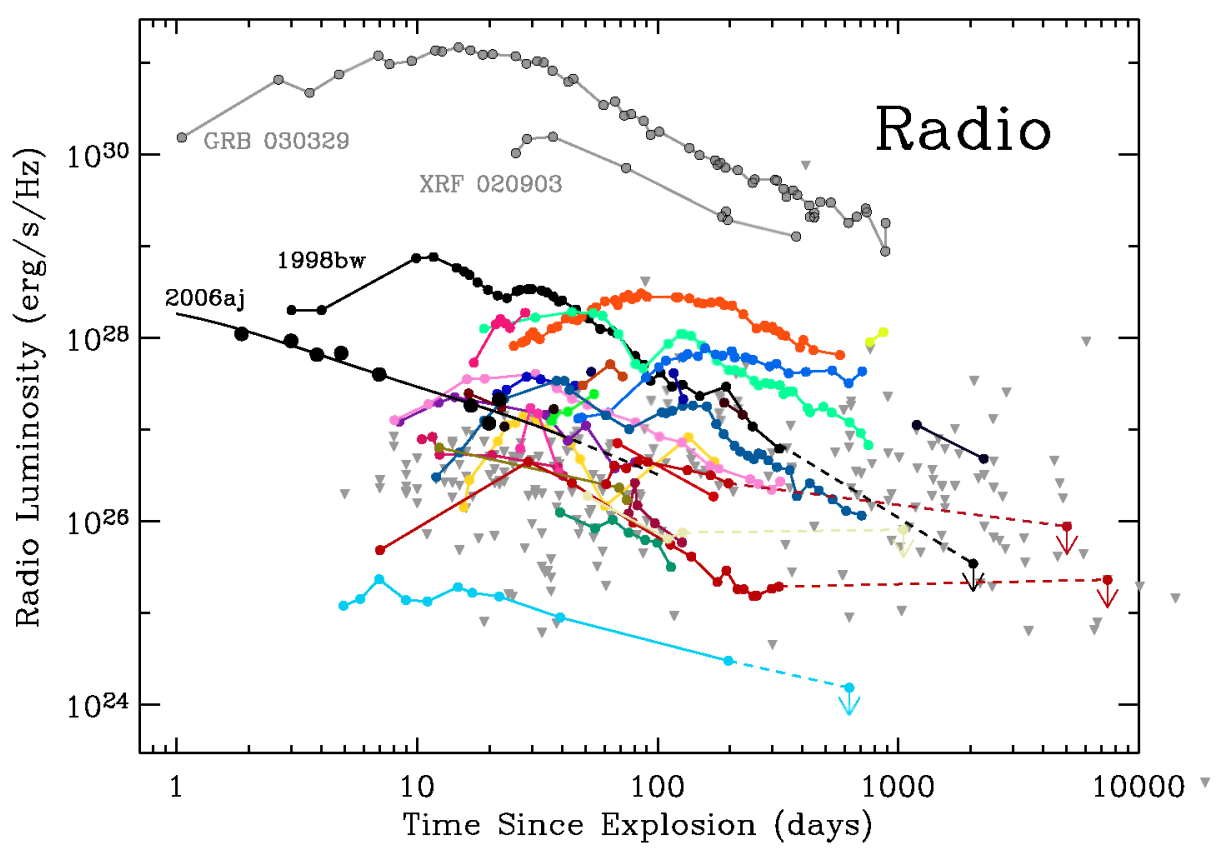

FIGURE 2. To date, 30 local $(d \lesssim 200 \mathrm{Mpc})$ SNe Ibc have been detected at radio wavelengths, the majority of which were found through my dedicated VLA survey. Detections are shown as colored lightcurves and $3 \sigma$ upper limits as inverted grey triangles. GRB-SN 1998bw and XRF-SN2006aj, also within the maximum distance of this sample, are distinguished by their bright early peaking radio emission (black). Both of these events were sub-energetic in comparison with typical GRBs (e.g., GRB 030329; grey) and XRFs (e.g., XRF 020903; grey).

\section{THE VELOCITY AND ENERGY OF THE FASTEST EJECTA}

As discussed by Chevalier [10], the radio emission from SNe Ibc is produced as the fastest ejecta shock-accelerate particles in the circumstellar medium (CSM). Turbulence amplifies the magnetic field and the accelerated (relativistic) electrons produce synchrotron radiation which peaks in the radio band just after the explosion. The spectral peak, defined by a low-frequency turn-over, is dominated by synchrotron self-absorption (SSA) as shown by our detailed studies of several SNe Ibc [11, 12]. This is different than the case for most Type II SNe which are dominated by external free-free absorption due to a dense CSM [13].

As shown by Readhead [14], for radio sources dominated by SSA the brightness temperature, $T_{B}=c^{2} F_{v} /\left(2 \pi k \theta^{2} v^{2}\right)$, is constrained to $5 \times 10^{10} \mathrm{~K}$, assuming the postshock energy density is in equipartition between magnetic fields, $\varepsilon_{B}$, and relativistic electrons, $\varepsilon_{e}$. With this assumption, robust constraints on the radius, $r$, and internal energy of the shocked CSM, $E_{i}$, are derived $[10,15,16]$. These quantities scale as simple 


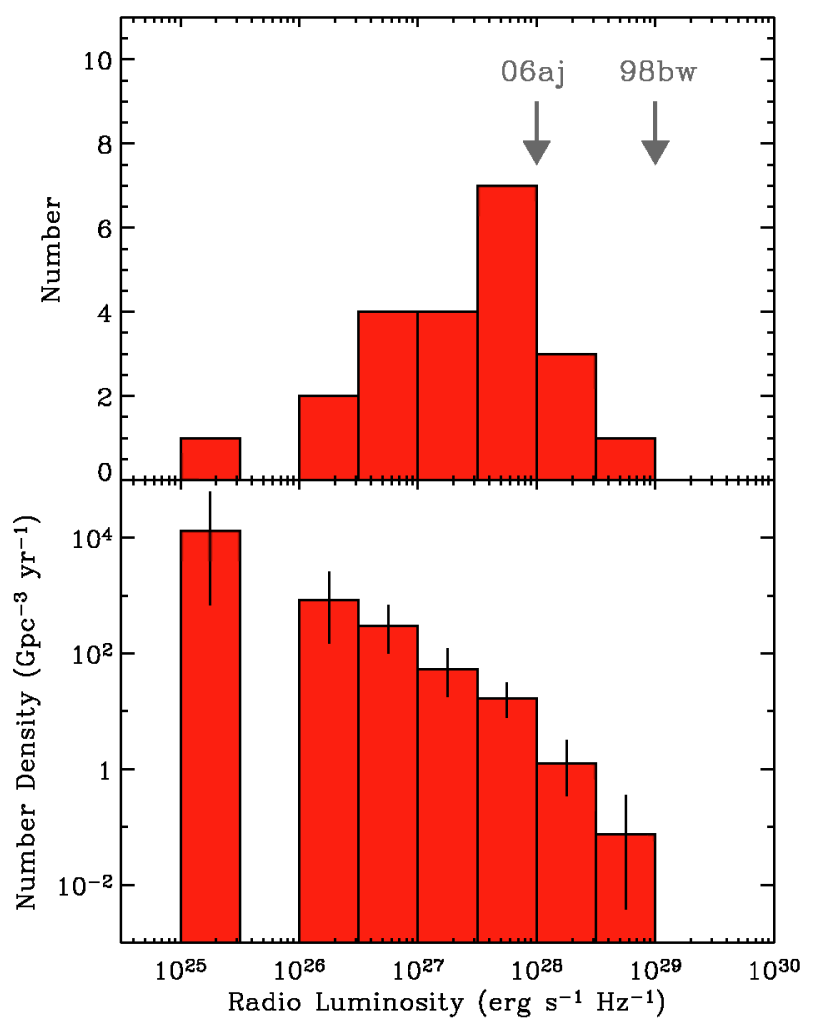

FIGURE 3. Top: The peak radio luminosity distribution for local SNe Ibc peaks near $L_{v} \approx$ $10^{28} \mathrm{erg} \mathrm{s}^{-1} \mathrm{~Hz}^{-1}$. The peak radio luminosities of GRB-SN 1998bw and XRF-SN 2006aj are shown for comparison (arrows) since they lie within a comparable distance as the optically-selected sample. Bottom: The radio luminosity function of SNe Ibc. Low luminosity SNe clearly dominate the intrinsic sample, though they are rarely observed since they fall below the current VLA detection limits. We emphasize that this analysis includes several biases, including effective monitoring time pre-2002 and efficiency of optical spectroscopic classification.

observables including the peak spectral frequency, $v_{p}$, the flux at the spectral peak, $f_{p}$, and the luminosity distance, $d$ (see Soderberg et al. 11, 12 for a detailed discussion). Here we adopt the simple model of Chevalier and Fransson 16, assuming $\varepsilon_{e}=\varepsilon_{B}=0.1$ which provides the following relations:

$$
\begin{gathered}
r=4 \times 10^{14}\left(f_{p} / \mathrm{mJy}\right)^{9 / 19}(d / \mathrm{Mpc})^{18 / 19}\left(v_{p} / 5 \mathrm{GHz}\right)^{-1} \mathrm{~cm} \\
E_{i}=1.5 \times 10^{41}(d / \mathrm{Mpc})^{8}\left(f_{p} / \mathrm{mJy}\right)^{4}\left(v_{p} / 5 \mathrm{GHz}\right)^{-7}\left(r / 10^{15} \mathrm{~cm}\right)^{-6} \mathrm{erg} .
\end{gathered}
$$

We note that the radius of the emitting material is only weakly dependent on the energy partition fractions, while departures from equipartition produce a strong increase the 


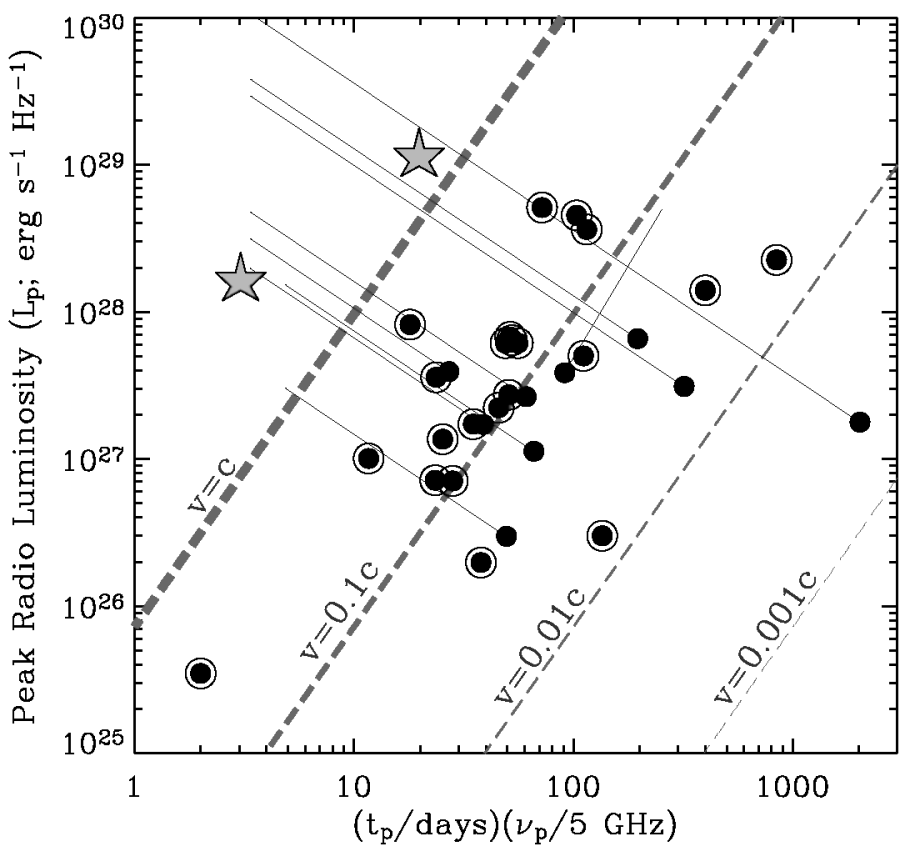

FIGURE 4. The peak radio luminosities of all the SNe Ibc detected to date are plotted against the timescale of peak. SNe with well-constrained peaks are shown as black encircled dots while those where the peak is not well constrained are shown as black dots with lines indicating the extrapolation of the radio light-curves between the range of typically observed peak times. GRB-SN 1998bw and XRF-SN 2006aj were also discovered within this volume and are shown as grey stars. Dashed lines indicate how the velocity of the fastest ejecta scales with the observed spectral parameters.

energy. Taken together with the observed peak time, $t_{p}$, the velocity of the shock is easily estimated from Equation 1 and the resulting values are shown for these SNe Ibc in Figure 4. SNe with early, bright radio emission have the fastest ejecta. As shown in the Figure, of the thirty radio bright SNe Ibc in this sample, the inferred velocities range from 0.01 to $0.5 \mathrm{c}$, and none show the mildly-relativistic ejecta inferred for GRBSN 1998bw [15] or XRF-SN 2006aj [5].

Next is a discussion of the explosion energetics. As derived by Chevalier [17], $E_{i}$ is just $\sim 20 \%$ of the total energy (kinetic and internal) of the shocked CSM, which in turn is equal to the kinetic energy of the fastest ejecta. Including this conversion factor, we find that the kinetic energy of the fastest ejecta, $E_{K} \sim 5 E_{i}$, spans $10^{46}-10^{49} \mathrm{erg}$ for $\mathrm{SNe}$ Ibc, a factor of $10^{2}$ to $10^{5}$ times less than that traced by the optical emission.

Finally, we compare these ejecta parameters (velocity, kinetic energy) with those of GRB and XRFs, including the class of sub-energetic bursts (Figure 5). We find evidence of a clear dichotomy between ordinary SNe Ibc and typical GRBs/XRFs: ordinary SNe couple roughly $10^{48}$ erg to their fastest (but non-relativistic) ejecta at $v \sim 0.15 c$. 


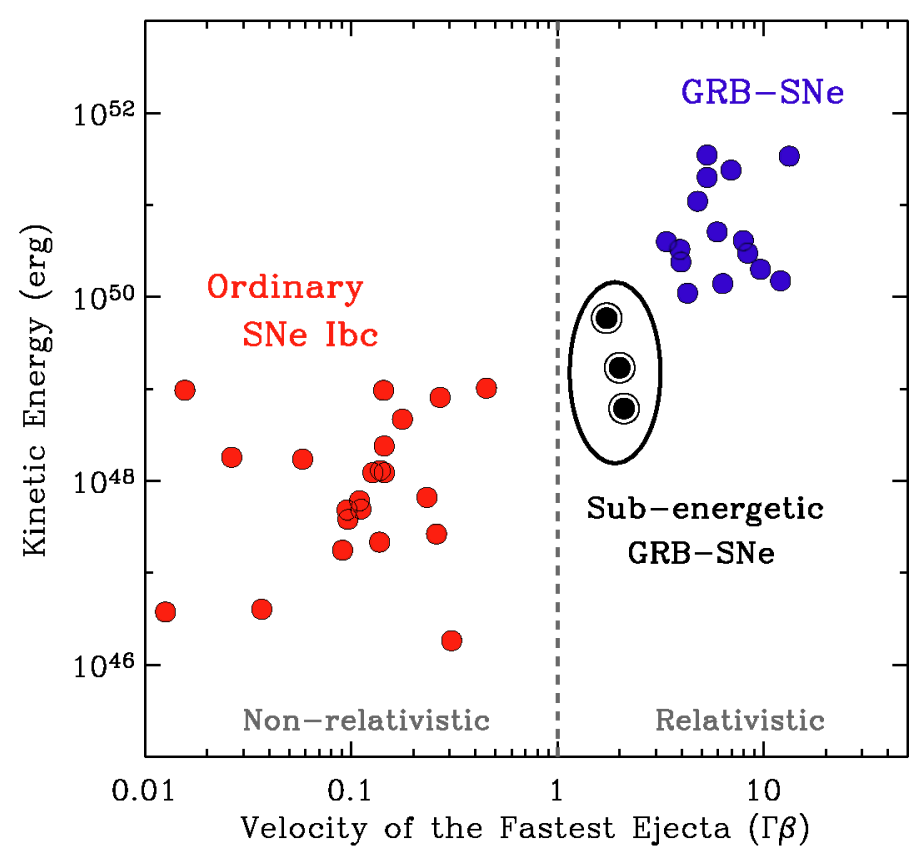

FIGURE 5. The kinetic energy and velocity of the fastest ejecta for radio bright $\mathrm{SNe}$ Ibc are compared with those of GRBs, XRFs, and sub-energetic bursts. Ordinary SNe Ibc (red) are distinguished in that they couple $10^{48}$ erg to non-relativistic ejecta at $v \approx 0.15 \mathrm{c}$ while GRBs and XRFs (blue) couple at $10^{51} \mathrm{erg}$ to relativistic material $(\Gamma \approx 10)$. Sub-energetic bursts (black) are intermediate between the two classes, coupling at least $10^{48}$ erg to mildly-relativistic $(\Gamma \approx 3)$.

Meanwhile, typical GRBs/XRFs couple $10^{51}$ erg to relativistic jets with $\Gamma \sim 10$. Subenergetic explosions bridge these two classes, coupling at least $10^{48}$ erg to mildlyrelativistic outflows with $\Gamma \sim 3$.

A related question is whether the observed radio emission for some $\mathrm{SNe}$ Ibc may be suppressed due to viewing angle effects, specifically important in the case of an associated GRB jet directed away from our line-of-sight [18]. In this scenario a rapid increase in radio emission is expected as the jet decelerates and spreads sideways, eventually crossing our line-of-sight on a timescale of $\sim 1$ yr after the explosion. As shown in Figure 2 and discussed in Soderberg et al. [8], radio observations of $\sim 70$ SNe Ibc taken between months to decades after the explosion reveal no evidence for associated GRB jets. Statistically we constrain the fraction of SNe Ibc harboring typical GRB jets to be less than $10 \%$. 


\section{CONCLUSIONS}

In conclusion, based on our large survey of optically selected local SNe Ibc, I find that (i) roughly $15 \%$ show detectable radio emission, (ii) the peak radio luminosities of $\mathrm{SNe}$ Ibc span four orders of magnitude with peak times between 1 and 1000 days, (iii) the kinetic energy and fastest velocity of these explosions are significantly different from those of typical GRBs and XRFs, (iv) compared with the sample of sub-energetic GRBs, $\lesssim 3 \%$ of SNe Ibc show ejecta properties indicative of an engine-driven explosion, and (v) the fraction of SNe Ibc harboring classical GRB jets viewed off-axis is constrained to less than $10 \%$.

Despite this progress, it remains an open question why just $\sim 1 \%$ of SNe Ibc give rise to GRBs or XRFs. A deeper understanding of the progenitors of ordinary SNe Ibc will shed light on this issue. With the recent advent of wide-field optical surveys, SNe Ibc from "blind" search campaigns will soon dominate new discoveries. Any investigation of the relation between the large-scale environment (host galaxy characteristics) and ejecta properties relies on SNe discovered through these unbiased surveys. In particular, host galaxy metallicity is argued to be a critical parameter as a proxy for the progenitor metallicity. Numerical models suggest that only low metallicity progenitors are able to produce the accretion-disk powered outflows inferred for GRBs [19]. Therefore, these models predict that engine-driven explosions are unlikely to be hosted in the luminous, high-metallicity galaxies monitored by targeted SN searches [20]. Looking forward, a radio survey focused exclusively on the SNe Ibc discovered through blind surveys will directly address and answer these crucial questions.

\section{REFERENCES}

1. S. E. Woosley, and J. S. Bloom, $A R A \& A 44,507-556$ (2006).

2. A. M. Soderberg, et al., ApJ 636, 391-399 (2006).

3. E. Pian, et al., Nature 442, 1011-1013 (2006).

4. A. M. Soderberg, et al., Nature 430, 648-650 (2004).

5. A. M. Soderberg, et al., Nature 442, 1014-1017 (2006),

6. E. Berger, S. R. Kulkarni, D. A. Frail, and A. M. Soderberg, ApJ 599, 408-418 (2003).

7. A. M. Soderberg, D. A. Frail, and M. H. Wieringa, $A p J L$ 607, L13-L16 (2004).

8. A. M. Soderberg, E. Nakar, E. Berger, and S. R. Kulkarni, ApJ 638, 930-937 (2006).

9. A. M. Soderberg, $A p J$ (2007), in preparation.

10. R. A. Chevalier, $A p J 499,810$ (1998).

11. A. M. Soderberg, et al., ApJ 621, 908-920 (2005).

12. A. M. Soderberg, R. A. Chevalier, S. R. Kulkarni, and D. A. Frail, ApJ 651, 1005-1018 (2006).

13. K. W. Weiler, N. Panagia, M. J. Montes, and R. A. Sramek, $A R A \& A$ 40, 387-438 (2002).

14. A. C. S. Readhead, $A p J$ 426, 51-59 (1994).

15. S. R. Kulkarni, et al., Nature 395, 663-669 (1998).

16. R. A. Chevalier, and C. Fransson, $A p J$ 651, 381-391 (2006).

17. R. A. Chevalier, $A p J$ 272, 765-772 (1983).

18. B. Paczynski, Acta Astronomica 51, 1-4 (2001).

19. S. E. Woosley, A. Heger, and T. A. Weaver, Reviews of Moderm Physics 74, 1015-1071 (2002).

20. K. Z. Stanek, et al., Acta Astronomica 56, 333-345 (2006). 